Psychological Medicine, 1985, 15, 3-8

Printed in Great Britain

\title{
EDITORIAL
}

\section{Biochemical hypotheses of premenstrual tension syndrome ${ }^{1}$}

As information has evolved concerning the incidence of cyclical emotional changes preceding the onset of menses, the premenstrual tension syndrome (PMT) has gained increased attention in both the lay and professional literature. Although there has been some disagreement as to the exact nature, the time of occurrence, or even the existence of PMT (Koeske, 1981), there is considerable consensus that the PMT syndrome indeed exists (Janowsky et al. 1966, 1967, 1969, 1973). Steiner et al. (1980) and Halbreich \& Endicott (1982) have now operationalized psychodiagnostic criteria for PMT which can allow separate studies to attain diagnostic consensus more accurately.

Several methodological problems in PMT research have limited exploration of how premenstrual physiology might influence mood. A major problem has been the strong reliance on the use of correlative techniques. Many hormones, electrolytes, neurotransmitters and somatic parameters change within the menstrual cycle, and a tradition has existed for assuming the aetiological importance of a favourite correlate which changes premenstrually. Although such correlations are a reasonable place to start, it may be naïve to believe that temporal correlations necessarily imply aetiology.

Another problem in PMT research is the general assumption that circulating ovarian-linked hormones as such are of fundamental significance in triggering and sustaining premenstrual emotional symptoms. It is equally possible that central neurotransmitters, neuromodulators, and neuroendocrine mechanisms control the onset and intensity of PMT, and that peripheral ovarian and other hormonal changes are merely simultaneous epiphenomena, and/or are driven by the above central influences, but do not actually exert behavioural effects.

The use of retrospective reports in PMT research has caused additional problems, with exaggerated incidences being reported, probably due to societal expectations (Koeske, 1981; Parlee, 1973; Sommer, 1978). In contrast, the incidence of PMT symptoms reported usually is relatively low in prospective PMT studies. Thus, many subjects who report PMT do not show symptoms in actual experiments. Also, since the intensity of PMT may vary from month to month (Green, 1982), such variation may introduce serious inconsistencies and variance problems into research studies. One recent step forward in this area has been the administration of repeated mood and behavioural ratings to prospective subjects over several cycles to screen for and document cyclic emotional changes before a given subject is used in a specific PMT study.

Also, there is some evidence that the stress of the experimental method itself may influence and confound studies exploring menstrual cycle-behavioural relationships (Koeske, 1981; Parlee, 1982). Conversely, a major impediment to understanding and treating PMT has been its remarkable sensitivity to treatment by placebo. Many promising aetiological hypotheses have been based on observed ameliorative effects in uncontrolled studies of compounds which have specific central biochemical effects. Subsequent controlled studies have shown these treatments to be no more effective than placebo.

There have been several hormonal hypotheses proposed to explain the aetiology of premenstrual tension. The most obvious of these involves an aetiological role for fluctuations in ovarian steroids. The oestrogen withdrawal hypothesis suggests that PMT is due to premenstrual oestrogen withdrawal. The observation that high levels of oestrogen have antidepressant effects (Klaiber $e t$ al. 1979) lends support to this possibility. However, the premenstrual fall in oestrogen is not the

1 Address for correspondence: Dr David S. Janowsky, Department of Psychiatry (M-003), University of California at San Diego, La Jolla, CA 92093, USA. 
only oestrogen decrease which occurs during the menstrual cycle. A large midcycle oestrogen peak occurs, followed by a decrease in oestrogen levels, and this withdrawal is not strongly associated with dysphoria. Furthermore, PMT has been shown to occur in postmenopausal and ovariectomized women in whom oestrogen levels are chronically low and fluctuate very little (Dalton, 1964).

Another oestrogen-related PMT hypothesis suggests that relative or absolute levels of oestrogen cause PMT. A report of PMT-like symptoms due to injected oestrogen (Morton, 1950) supports this possibility. Premenstrual tension, according to the oestrogen excess hypothesis, peaks at the time of the late luteal-premenstrual phase because of relatively high oestrogen levels, compared with rapidly falling progesterone levels. This possibility has received some biochemical support, in that women with premenstrual anxiety have been reported to have relatively high oestrogen/progesterone ratios (Backstrom \& Cartensen, 1974). However, a lack of PMT-like symptoms in normal women during the midcycle, when oestrogen levels peak and, likewise, in women with endogenously increased oestrogen levels, such as in metropathia haemorrhagia (Taylor, 1977), make an oestrogen excess hypothesis difficult to accept. The observation that PMT can occur in ovariectomized and in postmenopausal women, where oestrogen levels are relatively stable and are low, and the fact that one study has shown no alterations in oestrogen levels in PMT sufferers (Backstrom et al. 1983), are similarly not supportive.

Progesterone has also been considered in relation to PMT. Progesterone increases and subsequent decreases parallel menstrual cycle emotional changes (Janowsky et al. 1971), and the degree of loss of libido and depression in some women taking oral contraceptives has been positively correlated with the progestin content of the contraceptives (Grant \& Pryse Davies, 1968). Some evidence also suggests that low or rapidly falling levels of progesterone may be aetiological in premenstrual syndrome. Depressive symptoms have been observed to occur after withdrawal of exogeneous progesterone (Hamburg, 1966), and progesterone may be relatively lower in premenstrual tension sufferers prior to menstruation (Munday et al. 1977; Backstrom \& Cartensen, 1974; Smith, 1976), although a more recent study did not show altered progesterone levels in PMT sufferers (Backstrom et al. 1983). Finally, it is important to note that Backstrom et al. (1983) have observed that PMT does not appear to occur during anovulatory cycles, especially those associated with the lack of a luteal progesterone surge, although Adamopoulos et al. (1972) have made the opposite observation. Many clinics have employed progesterone as a treatment for premenstrual syndrome, with reported good results (Dalton, 1964). However, the efficacy of progesterone has not been shown to be better than placebo in at least one controlled study (Smith, 1976). Furthermore, PMT has been shown to occur in postmenopausal and ovariectomized women, in whom progesterone levels are presumably chronically low and relatively stable (Dalton, 1964).

Prolactin has also been suggested as being important in the aetiology of PMT. Prolactin levels probably fluctuate during the menstrual cycle, with ovulatory, mid-, and late luteal peaks (Tamura \& Igarashi, 1973; Vekemans et al. 1977); and increases in prolactin levels have been reported in PMT sufferers, as compared with controls (Kullander \& Svanberg, 1979). However, several reports do not show PMT sufferers to have greater absolute levels of prolactin during the premenstruum in comparison with controls (Andersch et al. 1978; Andersen \& Larsen, 1979; Munday et al. 1977). Furthermore, bromocriptine, a specific dopamine agonist which suppresses prolactin and which was shown initially to alleviate PMT, was not effective in later controlled studies (Green, 1982). Bromocriptine can improve mastodynia associated with premenstrual syndrome, but its effect on premenstrual dysphoria is much less obvious (Andersen et al. 1977; Graham et al. 1978). In any case, any beneficial effects of bromocriptine on the psychological symptoms occurring in PMT may be related not to prolactin changes but to direct effects on dopamine receptors.

One enduring hormonal hypothesis which we have explored is the fluctuation in the mineralocorticoid axis with premenstrual symptoms (Dalton, 1964; Janowsky et al. 1967, 1973). Premenstrual bloating and weight gain have led to speculations that increased sodium and water retention, possibly due to mineralocorticoids, may subtly shift salt and water balance in the central nervous system and thus cause emotional changes (Smith, 1976). There is also evidence that urinary sodium/potassium ratios fluctuate in parallel with negative affect during the menstrual cycle, possibly reflecting 
aldosterone or other mineralocorticoid effects (Janowsky et al. 1973; Demarchi \& Tong, 1972). Also, angiotensin-II and aldosterone have been shown to increase during the luteal phase, and aldosterone peaks premenstrually (Janowsky et al. 1969). However, PMT patients have not been shown to have consistently higher serum aldosterone levels when compared with normal controls. In support of an aldosterone hypothesis, evidence from a placebo controlled study suggests that the aldosterone antagonist and diuretic, spironolactone, may be effective in alleviating PMT, although further studies will be necessary to control for regression artefacts (Hendler, 1980; Simmons, 1983).

Another hypothesis suggests that PMT is due to fluctuations in adrenocortical hormones, such as androgens and glucocorticoids. Against this possibility is the observation that these hormones do not change dramatically during the menstrual cycle. Nevertheless, since cortisol may increase and androgens may decrease somewhat premenstrually (Vermeulen \& Verdonck, 1976; Walker \& McGilp, 1978), it is at least possible that an adrenal steroid could trigger central events, leading to the onset of PMT.

A logical strategy for studying PMT may be to assume that the same neurotransmitter and neuromodulators thought to regulate affective disorders are those which also modulate PMT. Although, as with PMT, the biochemical cause of affective disorders has not been discovered, the technology for exploring the biochemistry of mood is fairly advanced. Therefore, a strategy in which central neurochemicals and neuromodulators are evaluated as they relate to the menstrual cycle and its hormonal and parahormonal concomitants on the one hand, and to negative affect on the other hand, may be useful. Utilization of agonist and precursor techniques, neurotransmitters, antagonist administration, measurement of neurotransmitter and neuromodulator metabolites, and application of neuroendocrine strategies throughout the menstrual cycle in PMT sufferers may yield valuable information.

To this end, some evidence has emerged implicating changes in central neurotransmitters and neuromodulators in PMT (Rausch \& Janowsky, 1982), although this evidence is, as yet, by no means conclusive. The opioid polypeptides have been proposed as being causative as PMT (Reid \& Yen, 1981). This possibility is based on the observation that opioid polypeptides have dramatic effects on pituitary function, including blocking the release of luteinizing hormone. Since luteinizing hormone levels are minimal during the luteal phase, it has been suggested that excess endorphins may be present during this phase, and that PMT may be due to subsequent endogenous opioid withdrawal, possibly connected with rebound dopaminergic hyperactivity (Lal \& Puri, 1972). Against this hypothesis, however, is the observation that, in humans, beta-endorphin levels do not appear to change significantly during the luteal phase (Hamilton et al. 1982).

A current focus of our own work has been to consider cholinergic mechanisms in the regulation of PMT. In humans, acetylocholine has been proposed as a major modulator of both depression and stress (Janowsky et al. 1973, 1983), activating such disparate effects as pulse and blood pressure increases, negative affect, adrenal sympathetic amine release, and analgesia. In rats, ovarian hormone-acetylcholine interactions have been noted. Oestrogen induces an increase in muscarinic receptors in the ventromedial and anterior hypothalamic nuclei 72 hours after oestradiol injections in ovariectomized rats (Rainbow et al. 1980), and cholinergic muscarinic stimulation via cannulation of anterior hypothalamus (preoptic area) and mesencephalic reticular formation have been shown to facilitate an atropine-reversible sexual behaviour, lordosis, in the rat (Clemens et al. 1980).

Cholinergic interactions with the renin-angiotensin-aldosterone system and menstrually related remission of PMT symptoms in myasthenia gravis patients also contribute evidence for a role for acetylcholine in the aetiology of PMT (Rausch \& Janowsky, 1982). We have thus speculated that acetylcholine, acting alone and/or via noradrenergic, vasopressin, mineralocorticoid, opioid or other mechanisms, could regulate many of the events occurring in PMT (Janowsky et al. 1971, 1973; Rausch \& Janowsky, 1982).

Evidence also exists that catecholamine alterations may underlie PMT (Janowsky et al. 1971). Increases in endometrial monoamine oxidase (MAO) occur in the premenstrual phase of the cycle, and may be associated with a decrease in endogenous catecholamines premenstrually - a phenomenon consistent with a 'low catecholamine' hypothesis of affective disorders. Furthermore, a number of 
interactions have been demonstrated between the ovarian steroids and central norepinephrine activity, although it appears that these effects are quite time-dependent, and differ somewhat between experiments. For example, oestrogen in vitro was found to stimulate the efflux of dopamine and norepinephrine from rat brain synaptosones (Janowsky \& Davis, 1970). In contrast, oestrogen decreased dopamine release into hypophysial portal blood (Cramer et al. 1979) and decreased norepinephrine and dopamine efflux from rat brain slices (Vogel et al. 1970). Chronic oestrogen treatment has also been shown to reduce beta-adrenergic receptors in rat cortex (Wagner et al. 1979), an effect analogous to that noted with chronic antidepressant treatment.

Serotonin is another neurotransmitter which has been thought important in the aetiology of premenstrual tension. In humans, a reduced turnover of serotonin has been associated with impaired regulation of impulse control (Brown et al. 1983). Other studies in human subjects have shown a decreased tissue uptake and number of uptake sites for serotonin in clinically depressed patients (Rausch et al. 1981; Perry et al. 1983). A serotonin hypothesis of PMT is supported by observations of increases in brain serotonin uptake and turnover during progesterone and oestrogen treatment (Cone et al. 1981; Ladisich, 1977), and the possibility that rapid withdrawal of ovarian steroids could induce a rebound decrease in serotonin uptake and turnover, analogous to that described in major depression and impulse control disorders (Hackman et al. 1972). Associated with this hypothesis of PMT are observations that serotonin receptor concentrations and serotonin levels themselves decrease during proestrous in the female rat (Birgeon et al. 1980). Also, several animal studies have demonstrated an inhibitory role for serotonin in the regulation of sexual behaviour (Tagliamonte et al. 1969; Carter \& Davis, 1977).

We believe that many of the techniques utilized in studying the psychobiology of affective disorders and schizophrenia, as alluded to above, may prove useful in the understanding of the aetiology of PMT. Future research employing agonist/antagonist techniques may be among the most promising. To test an acetylcholine hypothesis, scopolamine or atropine should be tried in PMT. To test an opioid withdrawal hypothesis, naloxone should be given during the mid-luteal phase to induce PMT, and beta-endorphin or the opiates could be given during the premenstrual phase to see whether the symptoms are alleviated. Similarly, if a catecholamine depletion hypothesis has validity, a drug such as propranolol or alpha-methyl-para tyrosine (AMPT) should cause PMT in the mid-luteal phase, and drugs such as amphetamine, L-DOPA, and tyramine should have anti-PMT effects. If aldosterone and/or angiotensin are important in causing PMT, the aldosterone antagonist spironolactone should have consistent efficacy, and centrally acting angiotensin antagonists should also alleviate PMT. Likewise, if depleted serotonin is considered to be a cause of PMT, administration of tryptophan or 5-hydroxytryptophan, or a predominately serotonin reuptake inhibitor such as chlorimipramine, should have therapeutic efficacy in PMT; and serotonin blockers such as methysergide should precipitate the syndrome.

In addition to the importance of studying the role of neurotransmitters in the aetiology of PMT, there may be important clues to the aetiology of premenstrual tension in the observation that the symptoms of PMT seem to increase in proportion by life stresses (Dalton, 1964). It is possible that, through understanding the central biochemical modulation of stress and its interaction with cyclic changes in hormones and neurotransmitters, we may understand better the nature of PMT. To this end, it is important to note that changes in norepinephrine, opioids, GABA, serotonin, and acetylcholine all are perturbed during stressful situations, as may be menstrual cycle function (Janowsky et al. 1983; Dalton, 1964). Therefore, studies of the nature of changes in neurotransmitters and neuromodulators across the menstrual cycle under conditions of high and low stress may be especially helpful in understanding PMT.

Finally, it is important to focus on the effectiveness of placebo treatment in the attenuation of PMT. A major factor which has confounded PMT research and the development of effective treatments has been the potency of the placebo response. Only well-controlled trials should be considered indicative of an agent being therapeutic in the treatment of PMT. At another level, the profound and ameliorative 'placebo' effect on PMT may ultimately offer important clues and 
insights into the aetiology of PMT, for that which is altered by the placebo may be of fundamental importance in causing the PMT.

In conclusion, from the above remarks it is obvious that, although many hypotheses are promising, no existing hypothesis of PMT is even close to being proved at this time, either due to contradictory evidence or to the lack of completion of critical experiments. An important strategy for future research may involve the simultaneous study of the interactions of multiple neurotransmitter, neuromodulator, hormonal, environmental, and psychological factors over several menstrual cycles rather than the more traditional study of isolated factors. In any case, since the suffering, morbidity and mortality caused by PMT are considerable (Dalton, 1964), we feel that this syndrome represents a serious mental and public health problem, worthy of intensified and focused investigative efforts and support.

DAVID S. JANOWSKY AND JEFFREY RAUSCH

\section{REFERENCES}

Adamopoulos, D. A., Loraine, J. A., Lunn, S. F., Coppen, A. \& Daly, R. (1972). Endocrine profiles in premenstrual tension. Clinical Endocrinology 1, 282-292.

Andersch, B., Hahn, L., Andersson, M. \& Isaksson, B. (1978). Body water and weight in patients with premenstrual tension. British Journal of Obstetrics and Gynaecology 85, 546-551.

Andersen, A. N. \& Larsen, J. F. (1979). Bromocriptine in the treatment of the premenstrual syndrome. Drugs 17, 383-388.

Andersen, A. N., Larsen, J. F., Steenstrup, O. R., Svendstrup, B. \& Nielson, J. (1977). Effect of bromocriptine on the premenstrual syndrome. British Journal of Obstetrics and Gynaecology 84, 370-374.

Backstrom, T. \& Cartensen, H. (1974). Estrogen and progesterone in plasma in relation to premenstrual tension. Journal of Steroid Biochemistry 5, 257-260.

Backstrom, T., Sanders, D. \& Leask, R. (1983). Mood, sexuality, hormones and the menstrual cycle: II. Hormone levels and their relationship to the premenstrual syndrome. Psychosomatic Medicine 45, 503-507.

Biegeon, A., Bercovitz, H. \& Samuel, D. (1980). Serotonin receptor concentration during the estrus cycle in the rat. Brain Research 187, 221-225.

Brown, G. L., Goodwin, F. K. \& Bunney, W. E. (1983). Human aggression and suicide: their relationship to neuropsychiatric diagnoses and serotonin metabolism. Advances in Biochemical Psychopharmacology 34, 287-307.

Carter, S. C. \& Davis, J. M. (1977). Biogenic amines, reproductive hormones and female sexual behavior: a review. Behavior Reviews 1, 213-244.

Clemens, L. G., Humphreys, R. R. \& Dohanich, G. P. (1980) Cholinergic brain mechanisms and the hormonal regulation of female sexual behavior in the rat. Pharmacology, Biochemistry and Behavior 13, 81-88.

Cone, R. I., Davis, G. A. \& Coy, R. W. (1981). Effects of ovarian steroids on serotonin metabolism within grossly dissected and microdissected brain regions of the ovariectomized rat. Brain Research Bulletin 7, 639-644.

Cramer, O. M., Parker, C. R. \& Porter, J. C. (1979). Estrogen inhibition of dopamine release into hypophysial blood. Endocrinology 104, 419-421.

Dalton, K. (1964). The Premenstrual Syndrome. Heinemann: London.

Demarchi, G. W. \& Tong, J. E. (1972). Menstrual, diurnal, and activation effects on the resolution of temporally paired flashes. Psychophysiology 9, 362-367.

Graham, J. J., Harding, P. E., Wise, P. H. \& Berriman, H. (1978) Prolactin suppression in the treatment of premenstrual tension syndrome. Medical Journal of Australia 2 (3 suppl), 18-20.

Grant, E. C. G. \& Pryse Davies, J. (1968). Effect of oral contraceptives on depressive mood changes and on endometrial monoamine oxidase and phosphates. British Medical Journal iii, $777-780$.

Green, J. (1982). Recent trends in the treatment of premenstrual syndrome: a critical review. In Behavior and the Menstrual Cycle (ed. R. C. Friedman), pp. 367-395. Marcel Dekker: New York.

Hackman, E., Wirz-J ustice, A. \& Lichtsteiner, M. (1972). The uptake of dopamine and serotonin in the rat brain during progesterone decline. Psychopharmacologia 32, 183-191.

Halbreich, V. \& Endicott, J. (1982). Classification of premenstrual syndrome. In Behaviour and the Menstrual Cycle (ed. R. C. Friedman), pp. 243-265. Marcel Dekker: New York.

Hamburg, D. (1966). Effects of progesterone on behaviour. Research Publication-Association for Research in Nervous and Mental Disease 43, 251-265.

Hamilton, J. A., Aloi, J., Mucciardi, B. \& Murphy, D. L. (1982). Human plasma beta-endorphin through the menstrual cycle. Presented at the 21 st Annual Meeting of the American College of Neuropsychopharmacology. San Jan, Puerto Rico.

Hendler, N. H. (1980). Spironolactone for premenstrual syndrome. Female Patient 5, 17-19.

Janowsky, D. S. \& Davis, J. M. (1970). Progesterone-estrogen effects on uptake and release of norepinephrine by synaptosomes. Life Sciences 9, 525-531.

Janowsky, D. S., Gorney, R. \& Kelley, B. (1966). The curse vicissitudes and variations of the female fertility cycle: 1 . Psychiatric aspects. Psychosomatics 7, 242-247.

Janowsky, D. S., Gorney, R. \& Mandell, A. J. (1967). The menstrual cycle; psychiatric and ovarian-adrenocortical hormone correlates: case study and literature review. Archives of General Psychiatry 17, 459-469.

Janowsky, D. S., Gorney, R., Castelnuovo-Tedesco, P. \& Stone, C. B. (1969). Premenstrual-menstrual increases in psychiatric hospital admission rates. American Journal of Obstetrics and Gynecology $103(2), 189-191$.

Janowsky, D. S., Fann, W. E. \& Davis, J. M. (1971). Monoamines and ovarian hormone-linked sexual and emotional changes: a review. Archives of Sexual Behavior 1 (3), 205-218.

Janowsky, D. S., Berens, S. C. \& Davis, J. M. (1973). Correlations between mood, weight, and electrolytes during the menstrual cycle: a renin-angiotensin-aldosterone hypothesis of premenstrual tension. Psychosomatic Medicine 35 (2), 143-154.

Janowsky, D. S., Risch, S. C., Huey, L., Judd, L. \& Rausch, J. (1983). Central physostigmine-induced cardiovascular and behavioral changes: toward an acetylcholine hypothesis of stress. Psychopharmacology Bulletin 19 (4), 675-681.

Klaiber, E. L., Broverman, D. M., Vogel, W. \& Kobayshi, V. (1979) Estrogen therapy for severe persistent depressions in women. Archives of General Psychiatry 36, 550-554.

Koeske, R. D. (1981). Theoretical and conceptual complexities in the design and analysis of menstrual cycle research. In The Menstrual 
Cycle, Vol. 2: Research and Implications for Women's Health (ed. J. A. Noack and S. N. Elder), pp. 54-70. Springer-Verlag: New York.

Kullander, S. \& Svanberg, L. (1979). Bromocriptine treatment of the premenstrual syndrome. Acta Obstetrica Gynaecologica Scandinavica $58,375-378$.

Ladisich, W. (1977). Influence of progesterone on serotonin metabolism: a possible causal factor for mood changes. Psychoneuroendocrinology 2, 257-266.

Lal. H. \& Puri, S. K. (1972). Morphine withdrawal aggression: role of dopaminergic stimulation. In Drug Addiction: Experimental Pharmacology (ed. J. M. Singh, L. Miller and H. Lal), pp. 301-307. Futura Publishing Co.: Mount Kisco, New York.

Morton, J. H. (1950). Premenstrual tension. American Journal of Obstetrics and Gynaecology 60, 343-352.

Munday, M., Brush, M. G. \& Taylor, R. W. (1977). Progesterone and aldosterone levels in the premenstrual tension syndrome. Journal of Endrocrinology 73, $21 \mathrm{P}$.

Parlee, M. B. (1983). The premenstrual syndrome. Psychology Bulletin 80, 454-465.

Parlee, M. B. (1982). The psychology of the menstrual cycle: biological and physiology perspectives. In Behavior and the Menstrual Cycle (ed. R. C. Friedman), pp. 77-99. Marcel Dekker: New York.

Perry, E. K., Marshall, E. F. \& Blessed, G. (1983). Decreased imipramine binding in the brains of patients with depressive illness. British Journal of Psychiarry 142, 188-192.

Rainbow, T. C., DeGroff, G., Luine, V. N. \& McEwen, B. S. (1980). 17 beta estradiol increases the number of muscarinic receptors in hypothalamus nuclei. Brain Research 198, 239-243.

Rausch, J. L. \& Janowsky, D. C. (1982). Premenstrual tension: etiology. In Behavior and the Menstrual Cycle (ed. R. C. Friedman), pp. 397-427. Marcel Dekker: New York.

Rausch, J. L., Shah, N. S., Burch, E. A. \& Donald, A. G. (1981) Platelet serotonin uptake in depressed patients: circadian effect. Biological Psychiatry 17, 121-123.

Reid, R. L. \& Yen, S. S. C. (1981). Premenstrual syndrome. American Journal of Obstetrics and Gynaecology 139, 85-104.
Simmons, M. K. (1983), Possible new relief for premenstrual syndrome. Journal of the American Medical Association 250, $1371-1375$.

Smith, S. L. (1976). The menstrual cycle and mood disturbances. Clinical Obstetrics and Gynecology 19, 391-397.

Sommer, B. (1978). Stress and menstrual distress. Journal of Human Stress 4, 5-47.

Stciner, M., Haskett, R. F. \& Carroll, B. J. (1980). Premenstrual tension syndrome: the development of research diagnostic criteria and new rating scales. Acta Psychiatrica Scandinavica 62, 177-190.

Tagliamonte, A., Tagliamonte, P., Gessa, G. L. \& Brodies, B. B. (1969). Compulsive sexual activity induced by $\rho$-chlorophenylanine in normal and pinealectomized male rats. Science 166, 1433-1435.

Tamura, S. \& Igarashi, M. (1973). Serum prolactin levels during ovulatory menstrual cycle and menstrual disorders in women. Endocrinologica Japonica 20, 483-488.

Taylor, R.W. (1977). The treatment of premenstrual tension with dydrogesterone. Curremt Medical Research and Opinion 4 (Suppl. 4), 35-40.

Vekemans, M., Delroye, P., L'Hermite, M. \& Robyn, C. (1977). Serum prolactin levels during the menstrual cycle. Journal of Endocrinology and Metabolism 44, 989-993.

Vermeulen, A. \& Verdonck, L. (1976). Plasma androgen levels during the menstrual cycle. American Journal of Obstetrics and Gynecology $125,491-494$

Vogel, S. A., Janowsky, D. S. \& Davis, J. M. (1970). Effect of estradiol on stimulus-induced release of ${ }^{3} \mathrm{H}$-norepinephrine and ${ }^{3} \mathrm{H}$-serotonin from rat brain slices. Research Communications in Chemical Pathology and Pharmacology 1 (4), 451-459.

Wagner, H. R., Crutcher, K. A. \& Davis, J. N. (1979). Chronic estrogen treatment decreases beta-adrenergic responses in rat cerebral cortex. Brain Research 71, 147-151.

Walker, M. S. \& McGilp, S. (1978). Excretory patterns of urinary free II-hydroxycorticosteroids and total oestrogens throughout the normal menstrual cycle. Annals of Clinical Biochemistry 15, 201-202. 